\title{
A Global Score-Driven Beam Angle Optimization in IMRT
}

\author{
Humberto Rocha ${ }^{1,2}$, Joana M. Dias ${ }^{1,2}$, Tiago Ventura ${ }^{2,3,4}$, \\ Brígida C. Ferreira ${ }^{2,5}$, and Maria do Carmo Lopes ${ }^{2,3,4}$ \\ 1 CeBER and Faculdade de Economia, Universidade de Coimbra, \\ 3004-512 Coimbra, Portugal \\ 2 INESC-Coimbra, \\ 3030-290 Coimbra, Portugal \\ 3 Serviço de Física Médica, IPOC-FG, EPE, \\ 3000-075 Coimbra, Portugal \\ 4 Departamento de Física, Universidade de Aveiro \\ 3810-193 Aveiro, Portugal \\ 5 School for Allied Health Technologies, \\ 4400-330 Porto, Portugal \\ hrocha@mat.uc.pt, joana@fe.uc.pt, tiagoventura@ipocoimbra.min-saude.pt, \\ bcf@estsp.ipp.pt, mclopes@ipocoimbra.min-saude.pt
}

\begin{abstract}
Radiation therapy is one of the main treatment modalities for cancer. The objective of radiation therapy is to eliminate all cancer cells by delivering a prescribed dose of radiation to the tumor volume while sparing at the same time the surrounding tissues. Intensitymodulated radiation therapy (IMRT) is a sophisticated technologicallydriven type of radiation therapy where non-uniform radiation fields are used to irradiate the patient from different beam angle directions. Appropriate selection of beam irradiation directions - beam angle optimization (BAO) problem - enhance the quality of the treatment plan. The BAO problem is a very difficult global non-convex optimization problem for which there are few or none commercial solutions. Typically, the BAO procedure is driven by the outcome of the fluence map optimization (FMO) problem - the problem of calculating the most adequate radiation intensities. However, functions used for modeling the FMO problem have little clinical meaning. Typically, selection/validation of treatment plans is done considering a set of dosimetric measures. In this study, we propose a treatment plan global score, based on dosimetric criteria and its relative importance, as alternative plan's quality measure to drive the BAO procedure. For the clinical case of nasopharyngeal tumor, the use of a global score to drive the BAO procedure lead to higher quality treatment plans. For similar target coverage, an improved organ sparing was obtained.
\end{abstract}

Keywords: IMRT, Beam Angle Optimization, Global Score 


\section{Introduction}

Cancer numbers are expected to continue to increase with respect to its mortality and incidence features. More than half of the cancer patients will be treated with radiation therapy, either with curative intent or simply to palliate the symptoms. Radiation therapy aims to eliminate all cancer cells by delivering a prescribed dose of radiation to the cancerous tissues while sparing the surrounding healthy organs. Intensity-modulated radiation therapy (IMRT) is a sophisticated technologically-driven type of radiation therapy where the radiation beam is discretized into a set of small beamlets with different intensities by means of a multileaf collimator. Calculating the most adequate radiation intensities - fluence map optimization (FMO) problem - using this discretization of the radiation beam enhances an accurate control of the radiation doses received by different structures.

In IMRT, radiation is typically generated by a linear accelerator (linac) mounted on a gantry that can rotate along a central axis. Radiation beams from selected directions intersect the tumor, depositing in an additive way the total radiation dose in the tumor while trying to spare the surrounding organs that only receive radiation from a small subset of radiation beams. Typically, equispaced coplanar irradiation directions, i.e. evenly spaced directions laying on the rotation plane of the linac's gantry, are used in clinical practice. However, evidence shows that appropriate selection of beam irradiation directions - beam angle optimization (BAO) problem - can enhance the quality of the treatment plan [11]. Furthermore, for some types of cancers cases, e.g. intra-cranial tumors, the use of noncoplanar incidence directions improves substantially the treatment plan quality [5].

BAO and FMO problems can be solved separately, considering dosimetric surrogates or geometric features as quality measures of the beam ensembles [3,17]. Alternatively, BAO and FMO problems can be solved simultaneously and the optimal value of the FMO problem is used as quality measure of the beam ensembles $[2,10,18]$. Optimality and reliability is only granted by this later approach since beam angle directions for IMRT are often non-intuitive [23]. However, the objective functions used to drive the FMO problem are simple mathematical formulations with little or none clinical relevance. Thus, although the optimal FMO value is a beam ensemble score that is better correlated with treatment plan's quality than alternative dosimetric surrogates, it is far from being the ideal score. In this study, we propose BAO driven by a treatment plan global score as presented by Ventura et al. [24]. A clinical case of nasopharyngeal tumor, already treated at the Portuguese Institute of Oncology of Coimbra (IPOC), is used to acknowledge the performance of this novel approach. The remainder of the paper is organized as follows. Formulation of the BAO problem is presented in the next section. In section three we briefly describe the derivativefree optimization methods used to tackle the BAO problem. Computational tests are presented in section four followed by the conclusion's section. 


\section{Noncoplanar BAO in IMRT treatment planning}

The BAO problem can be generically divided into two distinct classes. In a first class, BAO is formulated as a combinatorial optimization problem, considering a discrete sample of all continuous beam angle directions. Since exhaustive searches are not feasible in terms of computational time, many different algorithms have been proposed, including gradient search [10], neighborhood search [2], simulated annealing [14], genetic algorithms [13], branch-and-prune [16], hybrid approaches [6] or iterative BAO [9]. Regardless of the algorithm used, it is not possible to calculate, in a polynomial run time, the optimal solution of the combinatorial BAO problem (NP hard problem) [4]. In a second class, a completely different methodological approach is considered. BAO is formulated as a continuous global optimization problem considering all possible beam angle directions around the tumor $[19,20,21,22]$. Here, a continuous formulation of the noncoplanar BAO problem is considered as described next.

\subsection{Noncoplanar BAO Model}

Let $n$ be the number of noncoplanar beam angle directions defined a priori by the treatment planner. Let $\phi$ denote the couch angle and $\theta$ denote the gantry angle. An unbounded formulation can be considered as angles $-5^{\circ}$ and $355^{\circ}$ or angles $365^{\circ}$ and $5^{\circ}$ are equivalent. While collisions between the patient/couch and the gantry never occur for coplanar optimization (for a fixed couch position at $\phi=0$ ), for some noncoplanar beam directions candidates collisions would occur. Thus, the choice of noncoplanar beam directions has collision restrictions. In order to maintain an unbounded formulation, collision restrictions are embedded in the objective function in the form of a penalty. A mathematical formulation for the noncoplanar BAO problem can then be obtained by considering a measure/score (objective function) such that the best beam ensemble corresponds to the function's minimum:

$$
\begin{aligned}
& \min f\left(\left(\theta_{1}, \phi_{1}\right), \ldots,\left(\theta_{n}, \phi_{n}\right)\right) \\
& \text { s.t. }\left(\left(\theta_{1}, \phi_{1}\right), \ldots,\left(\theta_{n}, \phi_{n}\right)\right) \in \mathbb{R}^{n} \times \mathbb{R}^{n} .
\end{aligned}
$$

In this study, two different objective functions $f\left(\left(\theta_{1}, \phi_{1}\right), \ldots,\left(\theta_{n}, \phi_{n}\right)\right)$ that measure the quality of a beam ensemble $\left(\theta_{1}, \phi_{1}\right), \ldots,\left(\theta_{n}, \phi_{n}\right)$ will be compared. The first, widely used, corresponds to the optimal value of the FMO problem for each fixed beam ensemble and incorporates a penalization for beam direction candidates where collision between the patient/couch and the gantry occur:

$$
f\left(\left(\theta_{1}, \phi_{1}\right), \ldots,\left(\theta_{n}, \phi_{n}\right)\right)= \begin{cases}+\infty & \text { if collisions occur } \\ \text { optimal value of the FMO } & \text { otherwise }\end{cases}
$$

The second, corresponds to a treatment plan's quality global score for each beam ensemble and it also incorporates a penalization for beam direction candidates 
where collision between the patient/couch and the gantry occur:

$$
f\left(\left(\theta_{1}, \phi_{1}\right), \ldots,\left(\theta_{n}, \phi_{n}\right)\right)= \begin{cases}+\infty & \text { if collisions occur } \\ \text { plan's quality global score } & \text { otherwise }\end{cases}
$$

FMO formulation/resolution and treatment plan's quality global score used to drive $\mathrm{BAO}$ are presented next.

\subsection{FMO formulation and resolution}

The FMO problem is usually formulated as a weighted sum function with conflicting objectives. Furthermore, constraints are often implemented as objectives, which difficult the trade-off between objectives without violating constraints. Thus, a multicriteria approach is the most suitable formulation for the FMO problem. Here, the FMO problem formulation considers an a priori multicriteria optimization approach based on a prescription called wish-list $[7,8,9]$.

Table 1 displays the wish-list constructed for the clinical case of nasopharyngeal tumor tested. Nasopharyngeal tumor cases are usually complex tumors to treat with radiotherapy. For simplicity, the organs at risk (OARs) in the wishlist are limited to the spinal cord, brainstem, parotids and oral cavity. For the nasopharyngeal case in study, two dose levels were defined for the planning target volume (PTV), tumor to be treated plus some safety margins: a higher dose level (70Gy) was defined for the tumor $\left(P T V_{70}\right)$ and a lower dose level (59.4Gy) was defined for the lymph nodes $\left(P T V_{59.4}\right)$. Several auxiliary structures were defined by computerized volume expansions to support the dose optimization. To prevent possible overirradiation in the lymph nodes, $P T V_{59.4}$ shell was created by removing a $10 \mathrm{~mm}$ margin of $P T V_{70}$ to $P T V_{59.4}$. To improve target coverage and conformity, two auxiliary ring shape structures, Ring $P T V_{70}$ and Ring $P T V_{59.4}$, were created with $10 \mathrm{~mm}$ of thickness at $10 \mathrm{~mm}$ distance from $P T V_{70}$ and $P T V_{59.4}$, respectively. External Ring, a ring of $10 \mathrm{~mm}$ thickness, was created next to the patient outer contour to prevent possible high values of dose entrance.

The wish-list contains 9 hard constraints and 10 prioritized objectives based on the prescribed/tolerance doses for the different structures included in the treatment planning optimization. All constraints are maximum-dose constraints and have to be strictly fulfilled. Objectives are optimized following a priority order defined a priori in the wish-list. Objectives with higher priorities are addressed first and thus are more likely to be fulfilled. For spinal cord and brainstem, organs whose functionality is jeopardized even if only a small subunit is damaged (serial organs), maximum-dose constraints are considered. For parotids, the larger salivary glands, and oral cavity, that contains the remaining salivary glands, mean-dose constraints are considered because the salivary glands are parallel type organs, i.e., organs whose function is not jeopardized if only a small portion is injured. 
Table 1. Wish-list for the nasopharyngeal tumor case.

\begin{tabular}{|c|c|c|c|c|c|c|}
\hline \multirow{3}{*}{ Constraints } & \multirow[b]{2}{*}{$\begin{array}{l}\text { Structure } \\
P T V_{59.4} \\
P T V_{70} \\
P T V_{59.4} \text { shell } \\
\text { Spinal cord } \\
\text { Brainstem } \\
\text { Ring } P T V_{59.4} \\
\text { Ring } P T V_{70} \\
\text { External Ring } \\
\text { Body }\end{array}$} & \multirow[b]{2}{*}{$\begin{array}{l}\text { Type } \\
\text { maximum } \\
\text { maximum } \\
\text { maximum } \\
\text { maximum } \\
\text { maximum } \\
\text { maximum } \\
\text { maximum } \\
\text { maximum } \\
\text { maximum }\end{array}$} & \multicolumn{4}{|c|}{ Limit } \\
\hline & & & \multicolumn{4}{|c|}{$\begin{array}{c}63.6 \mathrm{~Gy}(=107 \% \text { of prescribed dose }) \\
74.9 \mathrm{~Gy}(=107 \% \text { of prescribed dose }) \\
63.6 \mathrm{~Gy}(=107 \% \text { of prescribed dose }) \\
45 \mathrm{~Gy} \\
54 \mathrm{~Gy} \\
50.5 \mathrm{~Gy}(=85 \% \text { of prescribed dose }) \\
59.5 \mathrm{~Gy}(=85 \% \text { of prescribed dose }) \\
45 \mathrm{~Gy} \\
70 \mathrm{~Gy}\end{array}$} \\
\hline & Structure & Type & Priority & Goal & Parameters & Sufficient \\
\hline Objectives & $\begin{array}{l}P T V_{59.4} \\
P T V_{70} \\
P T V_{59.4} \text { shell } \\
\text { External ring } \\
\text { Spinal cord } \\
\text { Brainstem } \\
\text { Parotids } \\
\text { Oral cavity } \\
\text { Parotids } \\
\text { Oral cavity }\end{array}$ & $\begin{array}{l}\text { LTCP } \\
\text { LTCP } \\
\text { LTCP } \\
\text { maximum } \\
\text { maximum } \\
\text { maximum } \\
\text { mean } \\
\text { mean } \\
\text { mean } \\
\text { mean }\end{array}$ & $\begin{array}{c}1 \\
2 \\
3 \\
4 \\
5 \\
6 \\
7 \\
8 \\
9 \\
10\end{array}$ & $\begin{array}{c}1 \\
1 \\
1 \\
42.75 \mathrm{~Gy} \\
42.75 \mathrm{~Gy} \\
51.3 \mathrm{~Gy} \\
50 \mathrm{~Gy} \\
45 \mathrm{~Gy} \\
26 \mathrm{~Gy} \\
35 \mathrm{~Gy}\end{array}$ & $\begin{array}{c}T_{i}=59.4 \mathrm{~Gy} ; \alpha=0.75 \\
T_{i}=70 \mathrm{~Gy} ; \alpha=0.75 \\
T_{i}=59.4 \mathrm{~Gy} ; \alpha=0.75 \\
- \\
- \\
- \\
- \\
- \\
- \\
-\end{array}$ & $\begin{array}{l}0.5 \\
0.5 \\
0.5 \\
- \\
- \\
- \\
- \\
- \\
- \\
-\end{array}$ \\
\hline
\end{tabular}

The logarithmic tumor control probability $(L T C P)$ was considered for the target dose optimization [9],

$$
L T C P=\frac{1}{N_{T}} \sum_{l=1}^{N_{T}} e^{-\alpha\left(D_{i}-T_{i}\right)},
$$

where $N_{T}$ is the number of voxels in the PTV, $D_{i}$ is the dose in voxel $i, T_{i}$ is the prescribed dose, and $\alpha$ is the cell sensitivity parameter. LTCP penalizes doses lower than prescribed while the value slowly tends to zero for doses $D_{i}$ higher than the prescribed dose $T_{i}$. The ultimate goal is to obtain $L T C P=1$ corresponding to an homogeneous dose equal to $T_{i}$. An increase in the $\alpha$ value can improve the tumor coverage, i.e. the volume of the PTV that receives at least $95 \%$ of the prescribed dose.

The FMO problem formulated using the described wish-list was solved by a primal-dual interior-point algorithm, 2pєc [7], tailored for multicriteria IMRT treatment planning. This algorithm generates a single Pareto optimal IMRT plan, in an automated way, for a fixed number of beam directions [7]. The $2 p \epsilon c$ algorithm performs in two stages. In the first stage, all objectives are sequentially optimized, following the wish-list priorities, respecting the hard constraints. To assure flexibility for lower level objectives improvement, the optimization of tumor objectives $(L T C P)$ halts at a predefined sufficient value. After the optimization of each objective, a constraint is added to assure that the outcome of higher-order priorities are kept during the optimization of lower level priority objectives. At the end of the first stage, the treatment plan obtained fulfills all hard constraints of the wish-list as well as the goal for each objective or a higher value if the constraints prevent a better outcome. In the second stage, all objec- 
tives, except tumor $(L T C P)$ objectives, are fully optimized following the priority sequence of the wish-list. For more details on $2 p \epsilon c$ interior-point algorithm see Breedveld et al. [7].

The optimal value of the FMO problem is used to drive the BAO problem as a black-box function. Thus, the conclusions drawn considering the BAO problem coupled with this particular formulation/resolution of the FMO problem, are also valid if different formulations/resolutions are considered.

\subsection{Global Score for Treatment Plan Quality}

A Global Score $(G S)$ aiming to represent more accurately the overall quality of a treatment plan is proposed as alternative measure to guide the BAO procedure. Similarly to the treatment plan global score presented by Ventura et al. [24], GS is a weighted sum of individual scores assigned to each structure involved in the treatment planning optimization process:

$$
G S=\sum_{i} w_{i} \times \text { Score }_{i},
$$

where $w_{i}$ is the relative weight assigned to structure $i$ and $S c o r e_{i}$ is the score assigned to structure $i$. Thus, a relative weight must be assigned to each structure, based on its clinical relevance, and each structure's score, computed considering dosimetric goals typically inspected during treatment plan selection/validation, should express the fulfillment of the treatment prescription for that structure. Relative weights should be customized for each type of tumor in order to reflect the relative importance given by the radiation oncologist to the different planning objectives [24].

Clinical dose metrics typically used to verify organ sparing correspond to the maximum or mean tolerance doses, depending on the type of organ (serial or parallel, respectively). For tumor coverage, a clinical dose metric commonly used is the dose that $95 \%$ of the tumor volume receive $\left(D_{95}\right)$. Typically more than $95 \%$ of the prescribed dose is required. Table 2 depicts the prescribed and tolerance doses as well as the clinical dose metrics considered for the nasopharyngeal tumor case tested. The score for each structure corresponds to the ratio between clinical dose metrics and the corresponding planned doses. For the OARs, the score is given by

$$
\text { Score }_{O A R}=\frac{D_{P}}{D_{C}}
$$

where $D_{C}$ is the OAR clinical dose metric and $D_{P}$ the corresponding planned dose. For the PTVs, the score is given by

$$
\text { Score }_{P T V}=\frac{D_{C}}{D_{P}},
$$

where $D_{C}$ is the PTV clinical dose metric and $D_{P}$ the corresponding planned dose. Thus, a value of one is expected if the dose for that structure is equal to 
Table 2. Prescribed and tolerance doses for tumor volumes and OARs. Clinical dose metrics considered for plan's quality evaluation.

\begin{tabular}{lcccc}
\hline Structure & \multicolumn{2}{c}{ Tolerance Dose } & Prescribed & $\begin{array}{c}\text { Clinical } \\
\text { dose metrics }\end{array}$ \\
\cline { 2 - 4 } & Mean & Max & dose & dose \\
\hline PTV $_{70}$ & - & - & $70.0 \mathrm{~Gy}$ & $D_{95} \geq 66.5 \mathrm{~Gy}$ \\
PTV $_{\mathbf{5 9 . 4}}$ & - & - & $59.4 \mathrm{~Gy}$ & $D_{95} \geq 56.4 \mathrm{~Gy}$ \\
Brainstem & - & $54 \mathrm{~Gy}$ & - & $D_{\text {max }} \leq 54 \mathrm{~Gy}$ \\
Spinal cord & - & $45 \mathrm{~Gy}$ & - & $D_{\text {max }} \leq 45 \mathrm{~Gy}$ \\
Left parotid & $26 \mathrm{~Gy}$ & - & - & $D_{\text {mean }} \leq 26 \mathrm{~Gy}$ \\
Right parotid & $26 \mathrm{~Gy}$ & - & - & $D_{\text {mean }} \leq 26 \mathrm{~Gy}$ \\
Oral cavity & $45 \mathrm{~Gy}$ & - & - & $D_{\text {mean }} \leq 45 \mathrm{~Gy}$ \\
Body & - & $70 \mathrm{~Gy}$ & - & $D_{\text {max }} \leq 70 \mathrm{~Gy}$ \\
\hline
\end{tabular}

the respective clinical dose metric value. A score inferior to one is obtained for an improved target coverage or organ sparing. Overall, lower values of $G S$ imply treatment plans with better quality considering the metrics typically used to evaluate/compare treatment plans.

\section{Pattern Search Methods}

The highly non-convex nature of the noncoplanar BAO problem advises the selection of a derivative-free method. In previous works, we showed that a beam angle ensemble can be improved in a continuous manner using derivative-free algorithms. Pattern search methods (PSM) were selected for the resolution of the continuous BAO problem as they have the ability to avoid local entrapment and need a reduced number of function (FMO) evaluations to converge [19,20,21,22]. Each iteration of PSM have two steps with different purposes. In the first step, named search step, a global search is performed attempting to improve the outcome of the current best iterate. This global search is free of rules, except being finite, and can use any heuristic, strategy or method. If the first step fails, i.e. if the search step is empty or the procedure used was not able to improve the outcome of the current best iterate, the second step, named poll step, use the directions of positive bases to explore the neighborhood of the current best iterate. A positive basis is defined by a set of nonzero vectors (directions) that positively span the entire search space while no subset does. The main reason for using positive bases for optimization purposes is that at least one of its vectors (directions) can provide an improvement on the objective function value unless the current iterate is a stationary point. An example of a positive basis is the set of $2 n$ vectors $[I-I]$ where $I=\left[e_{1} \ldots e_{n}\right]$ is the identity matrix. In terms of $\mathrm{BAO}$, following each direction of this positive basis corresponds to the rotation of each beam direction clockwise and counter-clockwise for a certain amount (step-size) at each iteration. 
For a matter of computational time efficiency, the pattern search method implemented considers no trial points in the search step. The positive basis

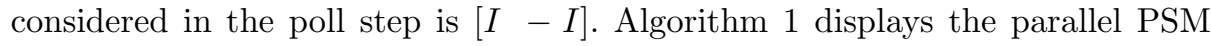
algorithm used.

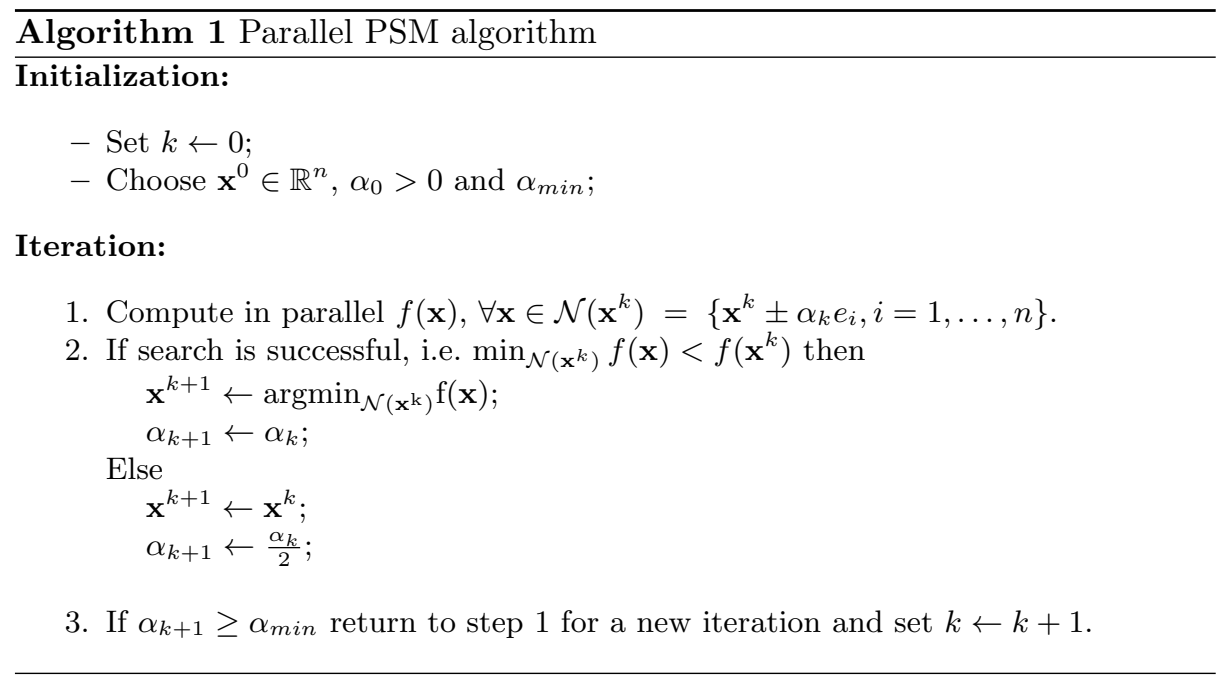

\section{Computational results}

Computational tests were performed on a Dell Precision T5600 with Intel Xeon processador $64 \mathrm{~GB}$ 1600MHz. YARTOS, an in-house optimization suite developed in MATLAB at Erasmus MC Cancer Institute in Rotterdam, was used to import DICOM images, optimize dose distributions and compute and visualize dose. YARTOS optimizer, $2 p \epsilon c$, was used to compute the optimal value of the FMO problem and thus to obtain the optimal fluences for a given beam direction ensemble.

The initial step-size considered by the implemented PSM algorithm was $\alpha^{0}=$ $2^{5}=32$ and the minimal value allowed was one, defining the stopping criteria. By choosing the initial step-size as a power of two, since step-size is halved at unsuccessful iterations, all beam directions considered are integer until the step-size becomes inferior to one which is the termination criteria.

Both the optimal value of the FMO problem and $G S$ were used to guide the PSM during the optimization of the noncoplanar BAO problem for a clinical case of nasopharyngeal tumor already treated at IPOC.

Treatment plans with seven equispaced coplanar beam directions are commonly used at IPOC to treat intra-cranial tumor cases. Therefore, treatment plans of seven noncoplanar beam directions were obtained using the optimal 
Table 3. Results of the beam angle optimization processes.

\begin{tabular}{lccccc}
\hline & \multicolumn{2}{c}{ Optimal FMO } & & \multicolumn{2}{c}{ Global Score } \\
\cline { 2 - 3 } \cline { 5 - 6 } & value & \% decrease & & value & $\%$ decrease \\
\hline$E q u i$ & 542.47 & - & & 0.924 & - \\
$B A O_{f}$ & 501.72 & $7.5 \%$ & & 0.899 & $2.5 \%$ \\
$B A O_{G S}$ & 502.25 & $7.4 \%$ & & 0.856 & $6.8 \%$ \\
\hline
\end{tabular}

value of the FMO problem $(f)$ and $G S$ to guide the PSM and were denoted $B A O_{f}$ and $B A O_{G S}$, respectively. These plans were compared with the typical seven-beam equispaced coplanar treatment plan denoted Equi. The objective of these comparisons is twofold. First, to compare the two measures of quality of a given beam ensemble. Second, to benchmark the noncoplanar results obtained with a coplanar plan typically used in clinical practice.

Table 3 depicts the results of the BAO processes both in terms of optimal FMO value and $G S . B A O_{f}$ treatment plans, obtained considering the optimal value of the FMO to guide the BAO procedure, achieve a $7.5 \%$ reduction of the optimal FMO value compared to Equi treatment plans. However, the improvement obtained in terms of $G S$ was only $2.5 \%$. On the other hand, $B A O_{G S}$ treatment plans, obtained considering $G S$ to guide the BAO procedure, achieve a $6.8 \%$ reduction of $G S$ value compared to Equi treatment plans which corresponded to a $7.4 \%$ reduction on the optimal FMO value. The history of the $\mathrm{BAO}$ processes considering the optimal FMO value and $G S$ value as objective functions are displayed in Fig. 1. Since PSM are non-increasing iterative methods, i.e. the next iterate only replaces the current one if its objective function value improves the best known, we can verify that the curves of the measures used to drive the BAO process are non-increasing. However, the behavior of the other measure is different meaning that an improvement in one measure do not necessarily imply an improvement in the other. In particular, improvements in the optimal FMO value have poor correspondence with similar improvements in the $G S$ value.

Regardless of objective function improvement, either optimal FMO value or $G S$ value, the quality of the results is typically acknowledged by different dose metrics. These dose metrics, considered for construction of $G S$, are displayed in Table 4 . By simple inspection it is possible to realize that $B A O_{G S}$ clearly outperforms both $B A O_{f}$ and Equi treatment plans. For similar target coverage, an enhance organ sparing is clearly obtained by $B A O_{G S}$ treatment plans. Comparison of the three treatment plans is straightforward using the graphical analysis proposed in SPIDERplan [24]. Fig. 2 displays customized radar plots that include the different structures considered for treatment planning optimization. The circular plotting area is divided into sections with an angular amplitude corresponding to the relative weight of the respective structure. Each structure's 

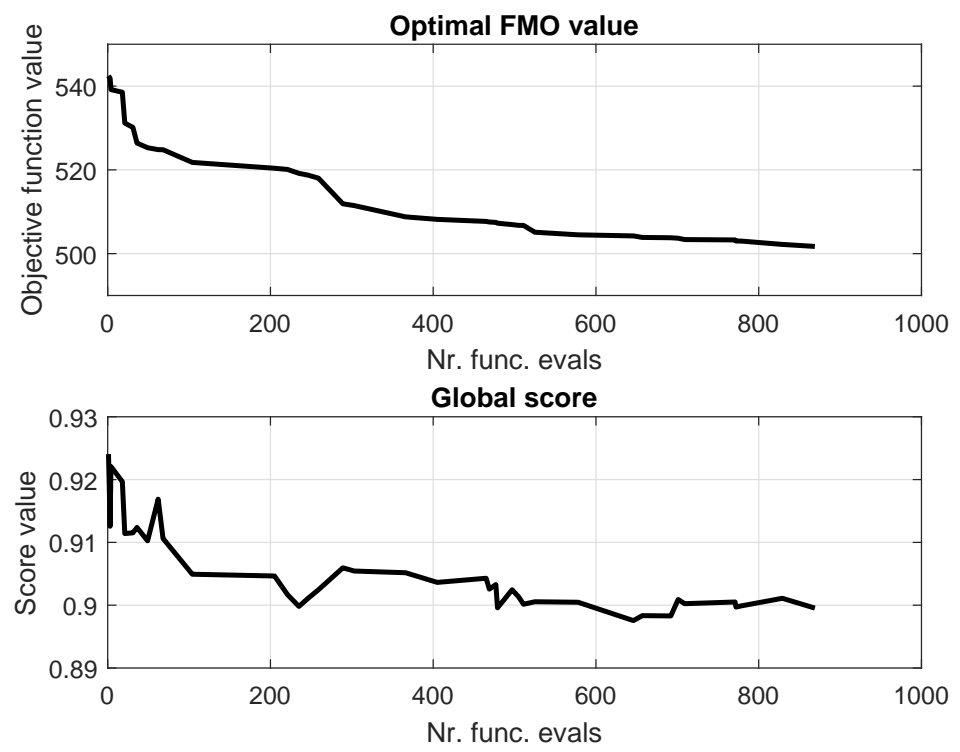

(a)
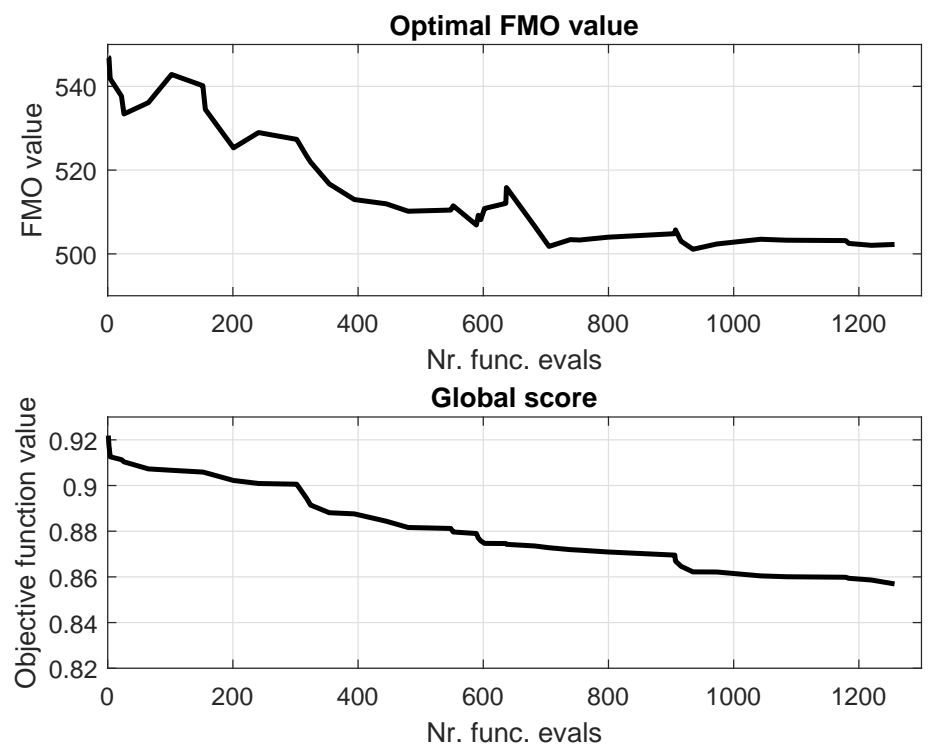

(b)

Fig. 1. History of the BAO procedure considering the optimal FMO value as objective function - 1(a) and considering $G S$ value as objective function - 1(b), respectively. 
Table 4. Target coverage and organ sparing obtained by treatment plans.

\begin{tabular}{|c|c|c|c|}
\hline Structure (Dose goal) & Equi & $B A O_{f}$ & $B A O_{G S}$ \\
\hline $\mathrm{PTV}_{70}$ (at $95 \%$ volume) & 65.4 Gy & $65.3 \mathrm{~Gy}$ & $65.4 \mathrm{~Gy}$ \\
\hline $\mathrm{PTV}_{59.4}$ (at $95 \%$ volume) & 56.2 Gy & $56.0 \mathrm{~Gy}$ & $56.0 \mathrm{~Gy}$ \\
\hline Brainstem (Max dose) & $42.6 \mathrm{~Gy}$ & 40.9 Gy & $35.3 \mathrm{~Gy}$ \\
\hline Spinal cord (Max dose) & $32.0 \mathrm{~Gy}$ & 34.7 Gy & $25.7 \mathrm{~Gy}$ \\
\hline Right parotid (Mean dose) & $21.3 \mathrm{~Gy}$ & 19.1 Gy & $18.4 \mathrm{~Gy}$ \\
\hline Left parotid (Mean dose) & $28.2 \mathrm{~Gy}$ & $22.3 \mathrm{~Gy}$ & $23.2 \mathrm{~Gy}$ \\
\hline Oral Cavity (Mean dose) & $32.2 \mathrm{~Gy}$ & $23.8 \mathrm{~Gy}$ & $21.4 \mathrm{~Gy}$ \\
\hline Body (Max dose) & $76.0 \mathrm{~Gy}$ & $77.2 \mathrm{~Gy}$ & $76.0 \mathrm{~Gy}$ \\
\hline
\end{tabular}

score is represented by a point on the bisector of the corresponding section that is exactly the score value away from the radar's center. The radar inner circle has unitary radius which corresponds to exactly meet the dosimetric goal defined for each structure. Optimal scores will converge to the radar plot center while increasing deviations from prescribed/tolerance doses will converge to the outer circle with radius equal to two. The polygon that connects all scores represents the quality of the treatment plan. The inner treatment plan, corresponding to $B A O_{G S}$ treatment plan, is easily identified as the best treatment plan.

In clinical practice, treatment plans are also typically compared using their cumulative dose-volume histograms (DVHs). Fig. 3 display the DVH results for the three treatment plan. The DVH curves also show that for similar target coverage, an improved organ sparing is obtained by $B A O_{G S}$ treatment plans. In particular, salivary glands are better spared which prevents xerostomia. This is a common complication of radiation therapy for head-and-neck cancer cases causing difficulties to swallow and decreasing the patient's quality of life. Thus, the enhanced salivary glands sparing is of the utmost interest.

\section{Conclusions and Future Work}

The BAO problem is a very difficult global non-convex optimization problem for which there are few or none commercial solutions. Given a beam ensemble with a corresponding optimal FMO value, typically a strategy or algorithm is used to find a beam ensemble with an improved FMO value. However, functions used for modeling the FMO problem have little clinical meaning and obtaining better function values do not necessarily imply that the corresponding treatment plan is preferred by the radiation oncologist. Typically, selection/validation of treatment plans is done considering a set of dosimetric measures whose relative importance depends on the tumor type, oncology center and even medical oncologist. Nevertheless, assuming that the decider's preferences (relative importance of the different, possibly conflicting, dosimetric criteria) can be established $a$ priori, it is possible to define a measure based on dosimetric criteria and its rel- 

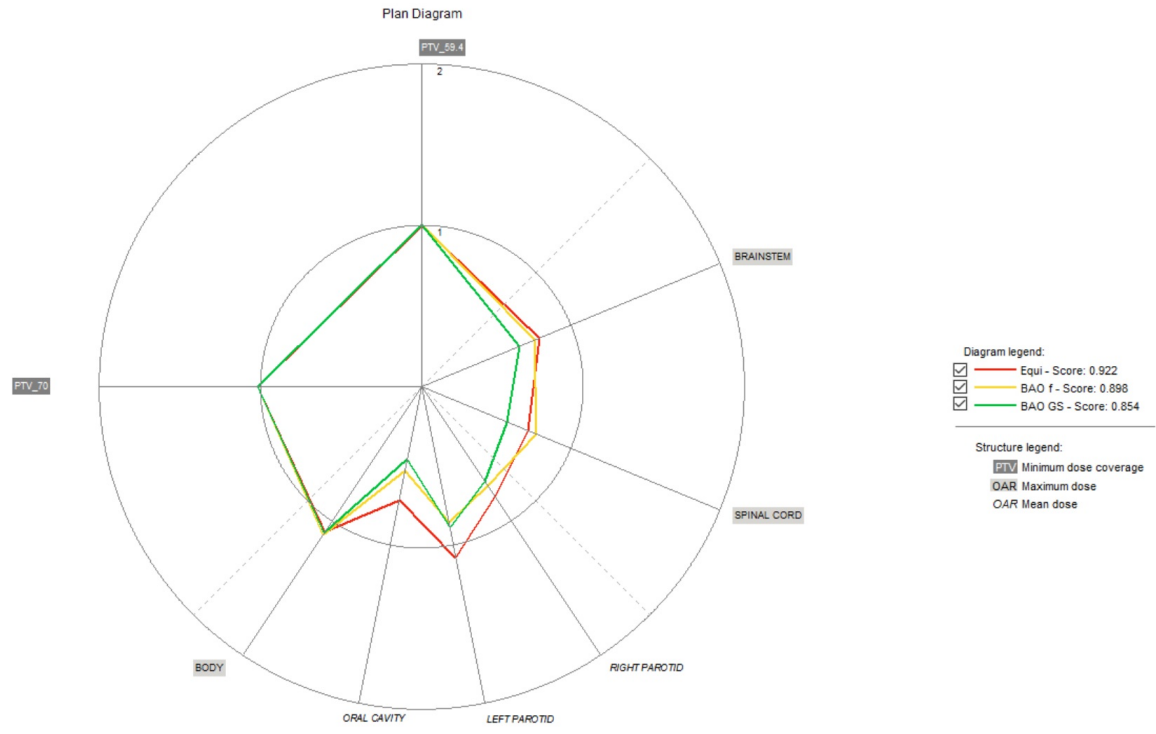

Fig. 2. Radar plots obtained by $B A O_{G S}, B A O_{f}$ and Equi.

ative importance that correlates better with the quality of the treatment plan. In this study, we propose a treatment plan global score, as presented by Ventura et al. [24], as alternative plan's quality measure to guide the BAO procedure.

For the clinical case of nasopharyngeal tumor tested, the use of $G S$ to drive the BAO procedure lead to higher quality treatment plans. For similar target coverage, an improved organ sparing is obtained by $B A O_{G S}$ treatment plans. It is important to highlight that BAO driven by $G S$ value obtained, as expected, a way better final $G S$ value than Equi treatment plan, but also lead to a final optimal FMO value similar to the obtained by BAO driven by the optimal FMO value. On the other hand, BAO driven by the optimal FMO value obtained, as expected, a way better final optimal FMO value than Equi treatment plan, but only a small improvement on $G S$ value compared to the obtained by BAO driven by $G S$. This result implies that improving the optimal FMO value do not necessarily improve $G S$, i.e. dose gains reflected in the optimal FMO value do not translate directly in dosimetric goals gains, while improving $G S$ correspond more often to a better optimal FMO value.

In future work, a $G S$ incorporating more structure's dosimetric measures, with weights clinically validated, should be further tested to acknowledge its advantage on driving a $\mathrm{BAO}$ procedure. Furthermore, one of the disadvantages of using the optimal FMO value to drive the BAO procedure is that the optimal beam ensemble found is jeopardized if a different fluence optimizer is used. It 


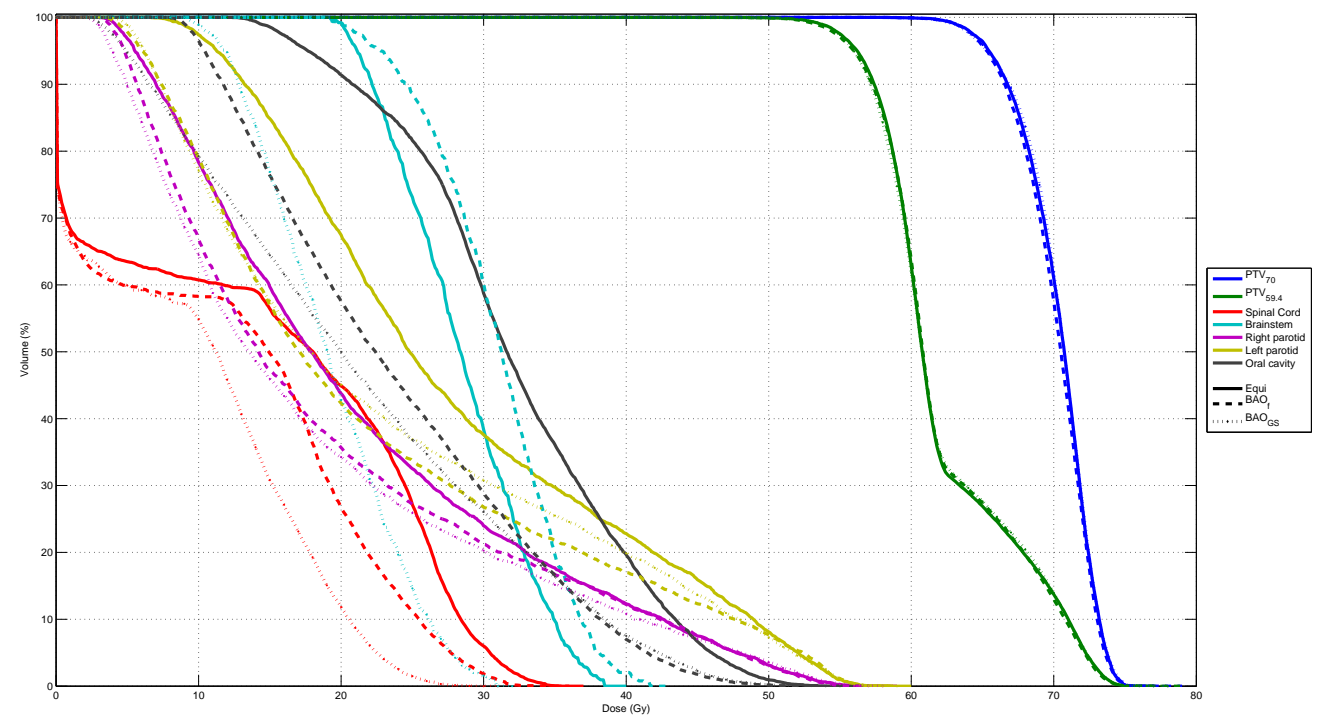

Fig. 3. Cumulative dose volume histogram comparing the results obtained by $B A O_{G S}$, $B A O_{f}$ and Equi.

is worth to test if BAO driven by $G S$ decreases the dependence on the fluence optimizer, i.e. if the optimal beam ensemble found using a given treatment planning system shows the same benefits when used in a different treatment planning system.

\section{Acknowledgements}

This work has been supported by the Fundação para a Ciência e a Tecnologia (FCT) under project grant UID/MULTI/00308/2013.

\section{References}

1. Alberto, P., Nogueira, F., Rocha, H., Vicente, L.N.: Pattern search methods for user-provided points: Application to molecular geometry problems. SIAM J. Optim. 14, 1216-1236 (2004)

2. Aleman, D.M., Kumar, A., Ahuja, R.K., Romeijn, H.E., Dempsey, J.F.: Neighborhood search approaches to beam orientation optimization in intensity modulated radiation therapy treatment planning. J. Global Optim. 42, 587-607 (2008)

3. Bangert, M., Oelfke, U.: Spherical cluster analysis for beam angle optimization in intensity-modulated radiation therapy treatment planning. Phys. Med. Biol. 55, 6023-6037 (2010) 
4. Bangert, M., Ziegenhein, P., Oelfke, U.: Characterizing the combinatorial beam angle selection problem. Phys. Med. Biol. 57, 6707-6723 (2012)

5. Bangert, M., Ziegenhein, P., Oelfke, U.: Comparison of beam angle selection strategies for intracranial imrt. Med. Phys. 40, 011716 (2013)

6. Bertsimas, D., Cacchiani, V., Craft, D., Nohadani, O.: A hybrid approach to beam angle optimization in intensity-modulated radiation therapy. Comput. Oper. Res. 40, 2187-2197 (2013)

7. Breedveld, S., Storchi, P., Keijzer, M., Heemink, A.W., Heijmen, B.: A novel approach to multi-criteria inverse planning for IMRT. Phys. Med. Biol. 52, 6339-6353 (2007)

8. Breedveld, S., Storchi, P., Heijmen, B.: The equivalence of multicriteria methods for radiotherapy plan optimization. Phys. Med. Biol. 54, 7199-7209 (2009)

9. Breedveld, S., Storchi, P., Voet, P., Heijmen, B.: iCycle: integrated, multicriterial beam angle, and profile optimization for generation of coplanar and noncoplanar IMRT plans. Med. Phys. 39, 951-963 (2012)

10. Craft, D.: Local beam angle optimization with linear programming and gradient search. Phys. Med. Biol. 52, 127-135 (2007)

11. Das, S.K., Marks, L.B.: Selection of coplanar or non coplanar beams using threedimensional optimization based on maximum beam separation and minimized nontarget irradiation. Int. J. Radiat. Oncol. Biol. Phys. 38, 643-655 (1997)

12. Davis, C.: Theory of positive linear dependence. Am. J. Math. 76, 733-746 (1954)

13. Dias, J., Rocha, H., Ferreira, B.C., Lopes, M.C.: A genetic algorithm with neural network fitness function evaluation for IMRT beam angle optimization. Cent. Eur. J. Oper. Res. 22, 431-455 (2014)

14. Dias, J., Rocha, H., Ferreira, B.C., Lopes, M.C.: Simulated annealing applied to IMRT beam angle optimization: A computational study. Physica Medica 31, 747$756(2015)$

15. Dias, J., Rocha, H., Ventura, T., Ferreira, B.C., Lopes, M.C.: Automated fluence map optimization based on fuzzy inference systems. Med. Phys. 43, 1083-1095 (2016)

16. Lim, G.J., Cao, W.: A two-phase method for selecting IMRT treatment beam angles: Branch-and-Prune and local neighborhood search. Eur. J. Oper. Res. 217, 609-618 (2012)

17. Llacer, J., Li, S., Agazaryan, N., Promberger, C., Solberg,T.D.: Noncoplanar automatic beam orientation selection in cranial IMRT: a practical methodology. Phys. Med. Biol. 54, 1337-1368 (2009)

18. Mišić, V.V., Aleman, D.M., Sharpe, M.B.: Neighborhood search approaches to noncoplanar beam orientation optimization for total marrow irradiation using IMRT. Eur. J. Oper. Res. 205, 522-527 (2010)

19. Rocha, H., Dias. J., Ferreira, B.C., Lopes, M.C.: Selection of intensity modulated radiation therapy treatment beam directions using radial basis functions within a pattern search methods framework. J. Glob. Optim. 57, 1065-89 (2013)

20. Rocha, H., Dias. J., Ferreira, B.C., Lopes, M.C.: Beam angle optimization for intensity-modulated radiation therapy using a guided pattern search method. Phys. Med. Biol. 58, 2939-53 (2013)

21. Rocha, H., Dias. J., Ferreira, B.C., Lopes, M.C.: Pattern search methods framework for beam angle optimization in radiotherapy design. Appl. Math. Comput. 219, 10853-65 (2013)

22. Rocha, H., Dias. J., Ventura, T., Ferreira, B.C., Lopes, M.C.: A derivative-free multistart framework for an automated noncoplanar beam angle optimization in IMRT. Med. Phys. 43, 5514-5526 (2016) 
23. Stein, J., Mohan, R., Wang, X.H., Bortfeld, T., Wu, Q., Preiser, K., Ling, C.C., Schlegel, W.: Number and orientation of beams in intensity-modulated radiation treatments. Med. Phys. 24, 149-160 (1997)

24. Ventura, T., Lopes, M. C., Ferreira, B., Khouri, L.: SPIDERplan: A tool to support decision-making in radiation therapy treatment plan assessment. Rep. Pract. Oncol. Radiother. 21, 508-516 (2016) 